\title{
The Relationship Between Exercise and Body Composition and Liver Enzymes
}

\author{
H. Bayram Temur*, Ramazan Ceylan \\ School of Physical Education and Sports, Bayburt University, \\ Bayburt, Turkey \\ Study Area: Bayburt, Turkey \\ Coordinates: $40^{\circ} 14^{\prime} 12$ ? N; $40^{\circ} 13^{\prime} 25$ ? E
}

Key words: Woman,BMI, HDL, LDL, TG, AST

\section{Introduction:}

It is known that regular physical activity benefits many systems, especially the cardiovascular system, by affecting lipid and lipoprotein levels. For example, as a result of regular activity, blood triglyceride and low-density lipoprotein are also thought to cause a decrease, while an increase in high-density lipoprotein level. But physical activity can be boring for some people. To do this regularly becomes even more difficult due to the boring and time concept and cannot be continued. It is precisely at this point that Zumba, one of the attractive activities, comes into play. Zumba combines both dance and sports to make physical activity more enjoyable. Zumba blends exercise movements and dance figures, allowing the activity to be enjoyed for a long time. Thus, it is estimated that it may cause a decrease in lipid level by spending high calories.

Exercise training positively affects lipid and carbohydrate metabolism, may result in moderate reductions in body weight, fat stores, total cholesterol and serum triglycerides, LDL cholesterol, and increases in antiatherogenic HDL cholesterol. Decreases initiated by exercise in total cholesterol and LDL cholesterol are highest when there are decreases in body weight (Tran \& Weltman, 1985).

Regular aerobic exercises can lead to moderate reductions in blood pressure, total blood cholesterol, serum triglycerides, low-density lipoprotein cholesterol, as

\section{Abstract}

Zumba exercise program applied for twelve weeks to know its effects on Body Mass Index (BMI), Body weight, High Density Lipoprotein (HDL), Low Density Lipoprotein (LDL), Triglyceride (TG) and Aspartate Aminotransferase (AST). A total of 15 women, whose average age was $40.33 \pm$ 9.15 years, were trained for one and a half hours for 3 days a week for 12 weeks. The blood samples were taken on an empty stomach four times before the training of Zumba and at the end of each week. Blood samples were taken 24 hours after exercise at the end of every four weeks. These blood samples were studied in a healthcare facility laboratory. The lengths and body weights of the participants were also determined. We found that there was a decrease in body weight and BMI measurement values and AST measurement values. However, no significant change in serum HDL, LDLand triglyceride values was evidenced.

well as increases in antiatherogenic high-density lipoprotein cholesterol in patients with hypertension (Tran \& Weltman, 1985; Franklin et al., 1991).

AST are enzymes that function in liver parenchymal cells and pass into the blood only in cell disorders. In acute heart muscle and skeletal muscle disorders, serum levels increase. AST, a cytoplasmic and mitochondrial enzyme, is found in the liver, heart muscle, skeletal muscle, kidney, brain, pancreas, lung, leukocyte, and erythrocytes (Öztürk, 2009).

In this study, it was aimed to investigate whether Zumba training performed regularly for 12 weeks affects body weight, BMI, serum HDL, LDL, TG, and AST values in women.

\section{Materials and Methods:}

In the study, 15 sedentary women with an average age of $40.33 \pm 9.15$ years were given Zumba training for one and a half hours three days a week for 12 weeks. No diet program was applied to the participants. Before the training program started, the height and bodyweight of the participants were determined along with their ages. In addition to these measurements, blood samples were taken to determine serum HDL, LDL, TG, and AST values. Blood samples were taken when participants were fasting in the morning. These measurements were repeated every four weeks. Blood samples taken from the participants were taken 24 hours later. The blood samples taken were sent to a healthcare 
facility laboratory without waiting. The data obtained were accumulated until the end of the training program. With the last measurements, the data were transferred to the computer environment for analysis. The statistical analyzes data were tabulated.

Length Length: It was measured using a meter while the participant was barefoot on a flat surface.

Body Weight: The participant was measured with a sports suit and an electronic precision scale while barefoot.

Statistical analysis; The data obtained were analyzed using the SPSS 21 package program using descriptive and repeated measures tests.

\section{Results:}

Table 1. Measurement Average Values for Variables During Exercise ( $\mathrm{N}=15$ common)

\begin{tabular}{lllll}
\hline Variable & 1. Mean+SD & 1. Mean+SD & 1. Mean+SD & 1. Mean+SD. \\
\hline Body wt. $(\mathrm{kg})$ & $81.13 \pm 2.83$ & $78.85 \pm 2.78$ & $77.47 \pm 2.92$ & $77.21 \pm 2.85$ \\
BMI $\left(\mathrm{kg} / \mathrm{m}^{2}\right)$ & $33.36 \pm 1.42$ & $32.40 \pm 1.38$ & $31.90 \pm 1.41$ & $31.60 \pm 1.38$ \\
HDL (mg/dL) & $44.96 \pm 11.12$ & $48.54 \pm 13.20$ & $43.61 \pm 8.82$ & $46.71 \pm 11.93$ \\
LDL (mg/dL) & $109.98 \pm 28.09$ & $108.74 \pm 25.93$ & $101.27 \pm 16.71$ & $103.96 \pm 24.70$ \\
Triglycerides & $112.79 \pm 49.09$ & $103.21 \pm 43.12$ & $103.57 \pm 55.67$ & $105.00 \pm 24.18$ \\
AST & $20.07 \pm 12.016$ & $13.64 \pm 4.069$ & $13.79 \pm 3.725$ & $14.07 \pm 4.582$ \\
\hline
\end{tabular}

Table-2: ANOVA : Measurement Points of Some Variables

\begin{tabular}{|c|c|c|c|c|}
\hline $\begin{array}{l}\text { Degisken } \\
\text { Source of Variance }\end{array}$ & Sum of squares & SD & Square Average & Sig. Diff. \\
\hline Bodyweight (kg) & \multicolumn{4}{|c|}{$(F=39.621 ; P=.000)$} \\
\hline Interview & 5846.779 & 13 & 449.752 & $1-2$. \\
\hline Measurement & 135.147 & 3 & $45 \cdot 049$ & $1-3$ \\
\hline Error & $44 \cdot 343$ & 39 & 1.137 & $1-4$ \\
\hline Total & 50324.9 & 26 & 55 & $2-3 . \quad 2-4$ \\
\hline \multicolumn{5}{|c|}{$\operatorname{BmI}\left(\mathrm{kg} / \mathrm{m}^{2}\right) \quad(\mathrm{F}=37.165 ; \mathrm{P}=.000)$} \\
\hline Interview & 1411.174 & 13 & $108.55^{2}$ & $1-2$. \\
\hline Measurement & 24.874 & 3 & 8.291 & $1-3$ \\
\hline Error & 8.701 & 39 & .223 & $1-4$ \\
\hline Total & 1444.749 & 55 & & $2-3 \cdot \quad 2-4$ \\
\hline $\mathrm{HDL}(\mathrm{mg} / \mathrm{dL})$ & $(\mathrm{F}+1.377$ & \multicolumn{2}{|c|}{$\mathrm{P}=.264)$} & \\
\hline Interview & 4913.221 & 13 & $377 \cdot 940$ & \\
\hline Measurement & 192.943 & 3 & $64 \cdot 314$ & \\
\hline Error & 1821.734 & 39 & 46.711 & \\
\hline Total & 6927.898 & 55 & & \\
\hline LDL (mg/dL) & \multicolumn{3}{|c|}{$(\mathrm{F}=.955 ; \quad \mathrm{P}=.423)$} & \\
\hline Interview & 21059.474 & 13 & 1619.960 & \\
\hline Measurement & 698.381 & 3 & 232.794 & \\
\hline Error & 9503.947 & 39 & 243.691 & \\
\hline Total & 31261.802 & 55 & & \\
\hline \multicolumn{2}{|c|}{ Triglycerides (mg/dL) } & \multicolumn{2}{|c|}{$(F=.642$} & \\
\hline \multicolumn{5}{|c|}{$\mathrm{P}=.593)$} \\
\hline Interview & 86196.857 & 13 & 6630.527 & \\
\hline Measurement & 848.714 & 3 & 282.905 & \\
\hline Error & 17193.286 & 39 & 440.853 & \\
\hline Total & 104238.857 & 55 & & \\
\hline AST (U/L) & $(\mathrm{F}=3.770$ & \multicolumn{2}{|c|}{$\mathrm{P}=.018)$} & \\
\hline Interview & 1131.857 & 13 & 87.066 & $1-2$. \\
\hline Measurement & 409.929 & 3 & 136.643 & $1-3$ \\
\hline Error & $1413 \cdot 571$ & 39 & 36.245 & $1-4$ \\
\hline Total & $2955 \cdot 357$ & 55 & & \\
\hline
\end{tabular}

As per Table-1 there is a decrease in body weight and BMI, LDL, TG and AST values compared to baseline values. HDL values were found to increase compared to the baselinevalue.

It was determined that there was a signif icant decrease in the bodyweight values of the participants. Similarly, a decrease in level was observed between BMI values. However, it was revealed that there was no significant difference between the measurements in HDL, LDL and Triglyceride values. Among AST measurement values, it was seen that there was a signif icant decrease.

\section{Discussion:}

The values of the three measurements taken during the training program were found to decrease in all measurement values. However, as a result of a statistical comparison of these values, it was determined that there was a decrease from the third measurement to the fourth measurement, but not statistically significant. In other measurements, it was revealed that there was a decrease of $p$ $<$ o.o1. Amano et al. (2001) practised obese individuals for three weeks a week for nine weeks. Again, Ribeiro (2004) with obese, found that continuous and interval swimming exercises, which are applied 5 days a week for 45 days a week for 12 weeks, have similar effects on weight loss.

The participants' mean BMI values' statistical result of comparison found no significant difference although there was only a decrease between the third and fourth measurements. A decrease of $\mathrm{p}<0.01$ was found in all of my other measurements. Amano et al. (2001) performed the exercises for obese men and women, whose average age was 41.6 years, for 12 weeks in a study of 30 minutes, 3 days a week. They reported a significant decrease in body mass indexes of the subjects. Nindl et al. (200o) applied the exercise program to 31 healthy women 5 days a week for 6 months. They stated that there was a $\mathbf{2 . 2} \%$ decrease in body mass at the end of the program.

The average HDL values of women before starting the training program was $44.96 \pm 11.12 \mathrm{mg} / \mathrm{dL}$, as a result of the four-week Zumba training, it was observed that this average value increased to $48.54 \pm 13.20 \mathrm{mg} / \mathrm{dL}$. At the end of the next four weeks, this mean value was found to drop to $43.61 \pm 8.82$ $\mathrm{mg} / \mathrm{dL}$. Finally, at the end of the training program, it was determined that this average value increased again. Although these average values increased overall, it was revealed that they were not statistically significant. Although the increase in the findings of the study was not significant, an increase of approximately $8 \%$ was detected. It was observed that there was an increase of approximately $4 \%$ between the first measurement values and the last measurement values. In the absence of these increases, it is thought that the participants may be dependent on the load levels in the nutrition and exercise program. In a study of 591 patients, they found a $5 \%$ increase in HDL cholesterol level (Maines, 1997). Hubinger et al. (1992) found a 
significant increase in serum HDL-C levels immediately after exercise, and a decrease of 15 minutes after exercise. In the study, although blood samples were taken 24 hours from the exercise, $4 \%$ and $8 \%$ increases were observed. Niebauer et al. (1996) also found that the increase in HDL was not significant in both women and men in a study of medium intensity and performed 3-4 times a week for 9 months. In their study, Tran et al. (1993) found that exercise increased serum HDL levels. In their study, Lavie \& Milani (1997) implemented 12-week exercise programs.They stated that HDL-K levels increased at the end of the study. Contrary to these kinds of literature, there are studies showing that exercise causes a decrease in HDL values (Kim et al., 2001; Wooten et al., 2008). Crouse et al. (1995) reported that these changes vary according to the intensity of physical condition and exercise. The potential mechanism for increases in HDL-K may be an increase in LPL activity. Lipoprotein lipase is related to the breakdown of triglycerides and provides the substrate for HDL production, several hours after exercise is metabolically active (Kantor et al., 1984).

The average LDL values of the subjects before the Zumba training program started was $109.98 \pm 28.09 \mathrm{mg} / \mathrm{dL}$ which constantly decreased at every week. Despite the decrease in these values, it was seen that it was not statistically significant. Although these values were not statistically significant, a decrease of approximately $8 \%$ was detected. This value was later decreased to $5.47 \%$. Tran \& Weltman (1985) revealed that exercise caused a 10.1\% reduction in LDL cholesterol. Again Niebauer et al. (1996) reported that LDL level decreased significantly in men and not changed in women in the study of moderate-intensity and performed 3-4 times a week for 9 months. It has been reported that moderate aerobic exercises (40-60\%) maxVO2 and endurance training reduce LDL cholesterol levels, while short-range exercises and training do not cause changes in blood lipid levels (Tamer, 1996). Hubinger et al. (1992) exercised 15 sedentary men for 30 minutes at 50 rpm and $60 \%$ of their maximal heart rate on a bicycle ergometer. As a result, they reported that no change in the LDL-C ratio was observed.

The mean of the triglyceride values measured before the training of the participants was $112.79 \pm 49.09 \mathrm{mg} / \mathrm{dL}$ which also decreased constantly but was not statistically significant. There was an $8.17 \%$ decrease between triglyceride levels between the first measurement and the third measurement. Tamer (1996) reported that TG levels decreased as a result of moderate aerobic exercises (40$60 \%) \mathrm{maxVO}_{2}$ and endurance training, and blood lipid levels did not change in short interval exercises and training. Tamer(1996) found that TG concentration did not change after endurance training. In their study, Lavie \& Milani (1997) stated that, at the end of the 12-week exercise program, especially triglycerides decreased. Niebauer et al.
(1996) also reported that the level of TG decreased significantly in men and did not change in women in a study of medium intensity and performed 3-4 times a week for 9 months. Sady et al. (1986) stated that decreases in triglyceride levels after exercise may occur with the effect of lipoprotein lipase (LPL), increase in TG cleavage and TG clearance. Hubinger et al. (1992) suggested that the level of triglycerides increased.

The average of AST values of the women included in the study was 20.07 $\pm 12.016 \mathrm{U} / \mathrm{L}$ before the exercise program. This value was found to be $13.64 \pm 4.069 \mathrm{U} / \mathrm{L}$ after the first four weeks, and subsequent measurements were 13.79 \pm $3.725 \mathrm{U} / \mathrm{L}$ and $14.07 \pm 4.582 \mathrm{U} / \mathrm{L}$, respectively. There was a statistically significant $(\mathrm{p}<0.05)$ decrease in these values. It was revealed that this significant decrease was between the first measurement and the last measurement, which is a decrease of approximately 30\%. Kim et al. (2001) after the loading of vitamin $\mathrm{C}$ in football players, AST levels were found to be lower than before loading. Literature data supports the findings of the study.

As a result, it was concluded that, by applying for different training programs, considering the other studies we have done on the subject, the exercise can have a positive effect on both body composition and liver enzymes, and the exercise habits, along with the type, intensity, duration of the exercise can be effective.

\section{References:}

Amano, M., Kanda, T. \& Maritani, T. (2001): Exercise Training and Autonomic Nervous System Activity in Obese Individuals. Med.Sci. Sports Exerc, 33(8):1287-1291,

Crouse, S.F., O’Brien, B.C. \& Rohack, J.J. (1995): Changes in Serum Lipids and Apoproteins After Exercise in Men With High Cholesterol: Influence of Intensity. J. Appl. Physiol., 79:279286.

Franklin, B.A., Gordon, S. \& Timmis, G.C. (1991): Exercise prescription for hypertensive patients. Ann. Med., 23:279-287.

Hubinger, .L.M. \& Mackinnon, L.T. (1992): The acute effects 3omin. Of moderate exercise on high-density lipoprotein cholesterol in untrained men. Eur.J. Appl. Physiol., 65(6):555560.

Kantor, M.A., Cullinane, E.M., Herbert, P.N. \& Thompson, P.D. (1984): Acute increase in lipoprotein lipase following prolonged exercise. Metabol., 33:454-457

Kim, J.R., Oberman, A., Setcher, G.F. \& Lee, J.Y. (2001): Effect of Exercise Intensity and Frequency on Lipid Levels in Man With Coronary Heart Disease: Training Level Comparison. Trial. Am.J. Cardiol., 87:942-946.

Lavie, C.J. \& Milani, R.V. (1997): Cardiac Rehabilitation. Brown DC, eds.Textbook of Cardiac Intensive Care. Pub. by: WB Saunders, Philadelphia

Maines, T.Y., Lavie, C.J., Milani, R.V., Cassidy, M.M., Gilliland, Y.E. \& Murgo, J.P. (1997): Effects of cardiac rehabilitation and exercise programs on exercise capacity, coronary risk factors, behaviour, and quality of life in patients with coronary artery

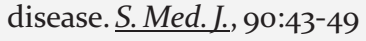


Niebauer, J., Hambrecht, R., Velich, T. Marburger, C., Hauer, K., Kreuzer, J., Zimmermann, R., Hodenberg, E., Schlierf, G., Schuler, G. \& Kübler, W. (1996): Predictive value of lipid profile for salutary coronary angiographic changes in patients on a low fat diet and physical exercise program. Am. J. Cardiol., 78:163-167.

Nindl, B.C., Harman, E.A. \& Marx, J.O. (200o): Regional body composition Changes in women after 6 months of periodized physical training. J. Appl. Physiol., 88(6):2251-2259.

Öztürk, Ç. (2009): Effects of Glycerol Supplement Before Acute Exercise OnSome Biochemical Parameters and Lactate and Aerobic Power in Athletes and Sedentary Individuals. Master Thesis, Selcuk University Institute of Health Sciences, Department of Physical Education and Sports Teaching, Konya:39; 1, 3, 5-10
Ribeiro, B.L., De Mello, M.A. \& Gobatto, C.A. (2004): Continuous and Intermittent Exercise: Effects of Training and Detraining on Body Fat in Obese Rats. Arch. Latinoam Nutr., 54(1):58-65.

Tamer, K. (1996): The effect of different aerobic training programs on you hormones, blood lipids and body fat percentage. Phys. Edu. Sports Sci.,1(1):1-11.

Tran, ZY. \& Weltman, A. (1985): Differential effects of exercise on serum lipid and lipoprotein levels are seen with changes in body weight: a meta-analysis. JAMA, 254:919-924

Wooten, J.S. Biggersta, K.D. \& Anderson, C. (2008): Response of Lipid, Lipoprotein-Cholesterol, and Electrophoretic Characteristics of Lipoproteins Following A Single Bout of Aerobic Exercise in Women. Eu. J. Appl. Physiol., 104:19-27. 